\title{
Aplicação do DMAIC para a melhoria contínua do sistema de estoque de uma empresa de informática
}

\author{
Bruno Braitt \\ Universidade Estadual de Santa Cruz - UESC \\ bbraitt@gmail.com \\ Diego de Castro Fettermann \\ Universidade Estadual de Santa Cruz - UESC \\ dcfettermann@gmail.com
}

\section{RESUMO}

Alinhado com o processo de melhoria contínua, o presente artigo propõe desenvolver um procedimento de melhoria contínua para o controle de insumos de embalagem utilizando o método DMAIC. A utilização de ferramentas aplicadas ao DMAIC e incorporadas ao método proposto possibilitou a coleta e análise dos dados, obtendo resultados que validaram a utilização do método para a melhoria contínua do processo. Os resultados obtidos estimam reduções de $36,09 \%$ das perdas relacionadas a pedidos parados e de $4 \%$ no custo com o gerenciamento do estoque.

Palavras-chave: melhoria contínua, fluxo de materiais, gerenciamento do estoque.

\section{ABSTRACT}

With the continuous process improvement, this article develops a continuous improvement procedure for inputs control in packaging process using the DMAIC method. The use of tools with the DMAIC enabled the data collection and analysis. The results validate the DMAIC use for continuous improvement process. The proposed reduced by $36.09 \%$ the stops orders and $4 \%$ costs with inventory management.

Keywords: continuous improvement, material flow, inventory management.

\section{Introdução}

Entre as características que dificultam o gerenciamento dos suprimentos em empresas de informática podem ser mencionados os curtos ciclos de vida dos produtos, a grande variedade de produtos, a baixa previsibilidade da demanda, a customização do produto durante o atendimento ao cliente (PARRA; PIRES, 2003). Além disso, a manutenção de baixos níveis de inventários e altos giros de estoques são variáveis importantes para competitividade das empresas deste setor (PARRA; PIRES, 2003). 
Diante disso, é indicada a aplicação de abordagens para melhoria de processos, como o método DMAIC (PEREZ-WILSON, 1999; ECKES, 2011; PANDE et al., 2001; WERKEMA, 2002; CARVALHO; PALADINI, 2005). Sua aplicação alinhada com a produção enxuta é frequente na literatura, como na redução de perdas por paradas não programadas em indústria moageira (SERVIN et al., 2012), na integração da manufatura enxuta e seis sigma (STEPHEN, 2004), em melhoria de processos na saúde (PROUDLOVE et al., 2008) ou em melhorias de processos de pequenas empresas (KUMAR et al., 2006), entre outros.

A partir disso, esse estudo tem por objetivo desenvolver um procedimento de melhoria contínua para o controle de insumos de embalagem utilizando o método DMAIC. Essa proposta é resultado da ocorrência de registros de paradas de produção por falta dos insumos, sendo este um importante motivo de perdas no processo, resultando em atrasos de faturamento e não atendimento das exigências dos clientes.

\section{Revisão de literatura}

O método DMAIC surgiu com a tarefa de reduzir variações, especialmente em processos de fabricação. O DMAIC possui funções similares aos seus antecessores na resolução de problema de fabricação, tais como o PDCA (DE MAST; LOKERBOOL, 2012). O DMAIC visa à melhoria do processo por meio da seleção correta de projetos e com etapas direcionadas para a solução de problemas dispostas de forma cíclica e contínua, contribuindo no processo de melhoria contínua. A integração de diversas ferramentas às fases do DMAIC contribui para estruturar um método sistemático e disciplinado, capaz de promover a redução da taxa de defeitos e falhas nos produtos e/ou serviços e/ou processos nas organizações (SANTOS, 2006; CARVALHO; PALADINI, 2005). Matos (2003) menciona que a abordagem passo-a-passo, definida através de etapas, a caracterização do problema e o entendimento das Características Críticas para a Qualidade (CTQ) são os principais fatores para o seu sucesso. A seguir, tem-se um breve detalhamento de cada etapa do DMAIC.

\subsection{Definir (Define)}

Nesta etapa deve-se identificar os processos críticos responsáveis pela geração de maus resultados, tais como: reclamações de clientes, altos custos de mão de obra, baixa qualidade de suprimentos, erros de forma, etc (CARVALHO; PALADINI, 2005). Nessa etapa recomenda-se a utilização da Carta de Projeto (Project Charter), documento formal que permite a realização de um estudo racional para o projeto (WERKEMA, 2013). Nesta carta é importante constar as informações sobre o núcleo que envolverá esclarecimentos sobre os resultados que estão sendo procurados, confirmando valor ao negócio, estabelecendo limites e recursos, comunicando metas e planos, e identificando os clientes e suas necessidades (LIN et al., 2013).

\subsection{Medir (Measure)}

Nesta etapa deve acontecer o refinamento e a focalização do problema (WERKEMA, 2013), desenvolvendo o levantamento dos dados históricos e análise do sistema de medição das variáveis de saída (MATOS, 2003). Nesta etapa, a coleta de dados é essencial para validar e quantificar o problema e/ou a oportunidade, objetivando a definição de prioridades e a tomada de decisões sobre os critérios que são necessários (LIN et al., 2013).

\subsection{Analisar (Analyze)}

Trata-se da etapa em que é realizada a identificação das variáveis que afetam o processo, sendo necessário encontrar as causas do problema para que se aprofunde nos detalhes, identificando a(s) atividade(s) críticas do mesmo (LIN et al., 2013). Além da análise 
dos dados coletados e da determinação das causas raízes de defeitos, também é possível identificar as diferenças entre o desempenho real e o planejado (SANTOS, 2006).

\subsection{Melhorar (Improve)}

Nesta etapa, determina-se a forma de intervenção para a redução do nível de defeitos do processos. Segundo Santos (2006), a garantia de melhoria do processo está associada a uma solução que seja capaz de eliminar e prevenir a ocorrência de problemas. Também é realizada a geração de ideias potenciais para a eliminação das causas fundamentais dos problemas, priorizados na etapa anterior (WERKEMA, 2002). Dentre ferramentas utilizadas nesta etapa estão DOE, FMEA, Brainstorming, 5W2H, entre outras (SATOLO et al., 2009; WERKEMA, 2013).

\subsection{Controlar (Control)}

A sustentabilidade da melhoria precisa de um sistema de controle para mantê-la dentro de intervalo de tolerância do processo. Nesta etapa, é confirmada a implantação da melhoria, a resolução do problema, a validação dos benefícios alcançados, as alterações necessárias aos procedimentos e instruções de trabalho, a implementação de ferramentas de controle e, por fim, a auditoria do processo e o monitoramento do desempenho (MATOS, 2003). Para esta etapa recomenda-se a utilização de CEP, histograma, técnicas de coleta de dados (SATOLO et al., 2009).

\section{Metodologia}

O método utilizado para melhoria do processo segue a sequência estruturada do DMAIC. O Quadro 1 apresenta as atividades, entradas, saídas e as ferramentas planejadas para cada etapa. 
Quadro 1 - Etapas e atividades para aplicação do DMAIC

\begin{tabular}{|c|c|c|c|c|}
\hline Etapas & Entradas & Atividades & Ferramentas & Saídas \\
\hline Define & $\begin{array}{l}\text { 1. Registro de paradas do setor de } \\
\text { Embalagem } \\
\text { 2. Registro de Não Conformidade - } \\
\text { Plano de Ação }\end{array}$ & $\begin{array}{l}\text { 1. Identificar o Problema } \\
\text { 2. Especificar o } \\
\text { problema } \\
\text { 3. Mensurar variáveis }\end{array}$ & $\begin{array}{l}\text { 1. Brainstorming } \\
\text { 2. Project Charter } \\
\text { 3. Análise } \\
\text { Econômica }\end{array}$ & $\begin{array}{l}\text { 1. Definição do } \\
\text { problema } \\
\text { 2. Escopo do projeto }\end{array}$ \\
\hline Measure & $\begin{array}{l}\text { 1. Registro de paradas do setor de } \\
\text { Embalagem } \\
\text { 2. Relatório das Programações de } \\
\text { Produção } \\
\text { 3. Registro de Valores ( } \mathrm{R} \$ \text { ) } \\
\text { Diários do Estoque de Insumos de } \\
\text { Embalagem }\end{array}$ & $\begin{array}{l}\text { 1. Coletar dados } \\
\text { 2. Integrar dados } \\
\text { 3. Confeccionar tabelas e } \\
\text { gráficos } \\
\text { 4. Mensurar perdas } \\
\text { econômicas }\end{array}$ & $\begin{array}{l}\text { 1. Estratificação } \\
\text { 2. Folha de } \\
\text { Verificação } \\
\text { 3. Histograma } \\
\text { 4. Gráfico de Pareto } \\
\text { 5. Análise } \\
\text { Econômica }\end{array}$ & $\begin{array}{l}\text { 1. Gráfico com } \\
\text { quantidade de OPs } \\
\text { paradas } \\
\text { 2. Gráfico com } \\
\text { quantidade e tipos de } \\
\text { insumos faltantes } \\
\text { 3. Gráfico Monetário } \\
\text { de OPs paradas } \\
\text { 4. Tabela de Custo de } \\
\text { aquisição das caixas }\end{array}$ \\
\hline Analyse & $\begin{array}{l}\text { 1. Gráfico com quantidade de OPs } \\
\text { paradas } \\
\text { 2. Gráfico com quantidade de } \\
\text { insumos faltantes } \\
\text { 3. Gráfico Monetário de OPs } \\
\text { paradas } \\
\text { 4. Tabela de Custo de aquisição } \\
\text { das caixas }\end{array}$ & $\begin{array}{l}\text { 1. Levantar possíveis } \\
\text { causas } \\
\text { 2. Investigar causa-raiz }\end{array}$ & $\begin{array}{l}\text { 1. Brainstorming } \\
\text { 2. Análise } \\
\text { Econômica } \\
\text { 3. Diagrama de } \\
\text { Causa e Efeito } \\
\text { 4. } 5 \text { Porquês }\end{array}$ & $\begin{array}{l}\text { 1. Causa raiz da falta de } \\
\text { insumos }\end{array}$ \\
\hline Improve & $\begin{array}{l}\text { 1. Causa raiz da falta de insumos } \\
\text { 2. Previsão de demanda das caixas } \\
\text { 3. Registro de Valores ( } \$ \text { ) } \\
\text { Diários do Estoque de Insumos de } \\
\text { Embalagem } \\
\text { 4. Custo de aquisição das caixas }\end{array}$ & $\begin{array}{l}\text { 1. Propor melhorias } \\
\text { 2. Propor ações } \\
\text { corretivas para o } \\
\text { problema } \\
\text { 3. Comparar cenário } \\
\text { atual com o do projeto de } \\
\text { melhorias }\end{array}$ & $\begin{array}{l}\text { 1. Brainstorming } \\
\text { 2. Método de } \\
\text { Previsão de } \\
\text { Demanda } \\
\text { 3. Análise } \\
\text { Econômica }\end{array}$ & 1. Proposta de melhoria \\
\hline Control & 1. Proposta de melhoria & $\begin{array}{l}\text { 1. Estabelecer } \\
\text { procedimento de } \\
\text { implementação e } \\
\text { acompanhamento }\end{array}$ & 1. $5 \mathrm{~W} 2 \mathrm{H}$ & $\begin{array}{l}\text { 1. Plano de Ação de } \\
\text { implementação }\end{array}$ \\
\hline
\end{tabular}

Fonte: O autor

\subsection{Apresentação da empresa}

A empresa está localizada no Pólo de Informática de Ilhéus e produz modelos de desktop, notebooks e netbooks. Além da unidade fabril também possui lojas próprias para a comercialização de seus produtos. A estratégia de vendas permite atender a vários tipos de clientes, desde pessoas físicas até editais de licitações de órgãos governamentais. Por conta disso produz computadores tanto com a logomarca da empresa quanto com logomarcas de clientes. Esta unidade possui diversas certificações da área da informática, além das certificações ISO 9001:2008 e ISO 14001:2004.

\section{Resultados}

\subsection{Etapa Define}

Os frequentes registros de paradas de produção e o registro de não conformidade para a falta de insumos de embalagem na Empresa foram o ponto de partida para o projeto. Para tanto, decidiu-se priorizar os insumos do tipo caixas, cuja falta resulta em grande impacto para o setor de Embalagem. A embalagem é o último estágio do processo de produção de computadores, sendo responsável por realizar a colagem de etiquetas de configuração e de componentes, além dos calços e das caixas, que facilitam o transporte e protegem os produtos. Apesar de um processo simples, suas paradas são responsáveis por perdas, resultantes dos atrasos de faturamento para empresa. São utilizadas cinco diferentes tipos de caixas para embalagem: tipo ' $\mathrm{H}$ ', 'N', 'S', 'PH' e 'A'. Uma análise de dados passados, 
correspondendo aos meses de Fevereiro, Março e Abril de 2013, constatou 173 Ordens de Produção (OP's) que ficaram paradas no setor aguardando a chegada de caixas para embalagens. $\mathrm{O}$ valor de todos os pedidos que ficaram parados nesse período e que possuíam OP's aguardando insumos de embalagem totalizaram o montante de $\mathrm{R} \$ 7.018 .359,41$. O Quadro 2 apresenta a Carta de Projeto (Project Charter), criada para formalizar o projeto de melhoria no processo de controle dos insumos de embalagem.

Quadro 2 - Carta de Projeto

\section{CARTA DE PROJETO}

\section{Redução das paradas de produção por falta de insumos de embalagem do tipo caixa}

\section{DESCRIÇÃO DO PROBLEMA}

$\mathrm{Na}$ fábrica de computadores da Empresa $\mathrm{A}$, as paradas no processo de embalagens por falta de insumos do tipo caixa foram apontadas como um dos problemas na rotina de trabalho, inviabilizando o cumprimento do planejamento das ordens de produção (OPs) e, consequentemente, gerando o registro de uma não conformidade (RNC). A empresa utiliza cinco diferentes tipos de caixas para embalar seus produtos, são eles: TIPO H, TIPO N, TIPO S, TIPO PH, TIPO A

Nos primeiros meses do ano de 2013, o valor médio mensal das perdas de produção decorrentes das paradas foi elevado, resultando em uma não disponibilização dos computadores para o Estoque de Produto Acabado e, posteriormente, impactando na expedição e entrega ao cliente.

Estas paradas de produção provocaram perdas econômicas e atrasos no faturamento da empresa em virtude das não entregas aos clientes nos prazos previstos e, também, aos custos operacionais para retrabalho dos computadores. Os pedidos parados no período sob análise totalizavam173 ordens de produção (OPs) paradas por falta de caixas, que somados representaram monetariamente $\mathrm{R} \$ 7.018 .359,4$ em faturamento para a Empresa A.

\section{META}

Reduzir as paradas de produção por falta de caixas para embalar os produtos por meio de melhorias de controle do insumo.

\section{AVALIAÇÃO DO HISTÓRICO DO PROBLEMA}

A partir do segundo mês de 2013, a fábrica da Empresa A teve um aumento de demanda não previsto com a antecedência necessária para que realizasse adequações aos pedidos dos insumos de embalagem do tipo caixa. A empresa não fazia uso de nenhum método de previsão de demanda, baseando-se apenas no histórico de pedidos e no controle das entradas e saídas do Estoque de Insumos. A forma como os pedidos eram feitos associada aos erros de inventários e valores incorretos de entrada e saída do estoque trouxeram consequências também para os meses de Março e Abril.

\section{RESTRIÇÕES E SUPOSIÇÕES}

Este projeto limita-se a apresentação de oportunidade de melhoria no controle de insumos de embalagem. Por isso, todos os gastos para implantação da solução a ser encontrada para o problema necessitarão de avaliação dos gestores envolvidos no processo de produção e da autorização da diretoria da empresa.

\section{CRONOGRAMA INICIAL}

1. DEFINIR - MARÇO/2013

2. MEDIR - ABRIL/2013

3. ANALISAR - MAIO/2013

4. MELHORAR - MAIO/2013

5. CONTROLAR - MAIO/2013 
O planejamento do levantamento de dados utilizou a ferramenta de estratificação, por meio da qual se estabeleceu os critérios abaixo:

a) Tempo - Mensurar o tempo de paradas no processamento das OP's por falta de caixas no período de Fevereiro, Março e Abril de 2013. Variável mensurada: dias de parada.

b) Tipo - Mensurar a quantidade de ocorrências de falta de cada um dos cinco tipos de caixas que resultaram em OP's paradas. Variável mensurada: Quantidade de OPs paradas por falta de caixas.

c) Tipo - Mensurar o valor monetário de cada OP parada na produção aguardando a disponibilidade de insumos de embalagem. Variável mensurada: Valor em reais (R\$) das OP's

d) Tipo - nível de estoque do insumo de embalagem durante o período de Fevereiro, Março e Abril de 2013. Variável mensurada: Quantidade de unidades do estoque de cada um dos cinco tipos de caixas.

e) Tipo: Mensurar o valor de cada um dos cinco tipos de caixas utilizadas no processo de embalagem. Variável mensurada: Valor unitário em reais $(\mathrm{R} \$)$ de cada tipo de caixa.

O levantamento de dados iniciou-se pelos registros dos impactos de produção, identificando somente as paradas relacionadas à falta de caixas para o período. $\mathrm{O}$ levantamento desses dados foi realizado por meio da folha de verificação e apresentado de forma parcial para as embalagens mais críticas (tipo H, A e PH) (QUADRO 3).

\section{Quadro 3 - Folha de Verificação parcial utilizada para a coleta de dados}

\begin{tabular}{|c|c|c|c|c|c|}
\hline \multicolumn{6}{|c|}{ FOLHA DE VERIFICAÇÃO } \\
\hline \multicolumn{6}{|c|}{ Área: Produção } \\
\hline \multicolumn{6}{|c|}{ Fatores de estratificação: tipo de caixa, mês, número da OP, qtd de produtos, valor do pedido, tempo parado (em dias) } \\
\hline \multicolumn{6}{|c|}{ Período:fev/13 a abril/2013 } \\
\hline $\begin{array}{l}\text { Tipo de } \\
\text { Caixa }\end{array}$ & Período & $\mathbf{N}^{\circ} \mathbf{O P}$ & $\begin{array}{c}\text { Quantidade de } \\
\text { Produtos (unidades) } \\
\end{array}$ & Valor do Pedido & $\begin{array}{l}\text { Tempo decorrido: data de produção } \\
\text { e data de finalização do processo (dias) }\end{array}$ \\
\hline \multirow{7}{*}{$\begin{array}{l}x \\
0 \\
0\end{array}$} & FEVEREIRO & $* * 187$ & 100 & $\mathrm{R} \$ 119.912,00$ & 19 \\
\hline & FEVEREIRO & $* * 249$ & 10 & $\mathrm{R} \$ 14.160,10$ & 15 \\
\hline & FEVEREIRO & $* * 299$ & 20 & $\mathrm{R} \$ 37.740,00$ & 14 \\
\hline & FEVEREIRO & $* * 397$ & 5 & $\mathrm{R} \$ 7.516,00$ & 9 \\
\hline & FEVEREIRO & $* * 401$ & 1 & $\mathrm{R} \$ 1.548,76$ & 8 \\
\hline & MARÇO & $* * 471$ & 2 & $\mathrm{R} \$ 4.845,00$ & 5 \\
\hline & MARÇO & $* * 475$ & 2 & $\mathrm{R} \$ 4.845,00$ & 5 \\
\hline \multirow{11}{*}{$\begin{array}{l}\varangle \\
\varrho\end{array}$} & MARÇO & $* * 694$ & 20 & $\mathrm{R} \$ 20.245,60$ & 5 \\
\hline & MARÇO & $* * 693$ & 50 & $\mathrm{R} \$ 67.064,00$ & 5 \\
\hline & MARÇO & $* * 692$ & 100 & $\mathrm{R} \$ 119.128,00$ & 3 \\
\hline & MARÇO & $* * 756$ & 220 & $\mathrm{R} \$ 114.681,60$ & 3 \\
\hline & MARÇO & $* * 690$ & 250 & $\mathrm{R} \$ 130.320,00$ & 3 \\
\hline & MARÇO & $* * 672$ & 50 & $\mathrm{R} \$ 73.564,00$ & 2 \\
\hline & MARÇO & $* * 574$ & 100 & $\mathrm{R} \$ 184.628,00$ & 2 \\
\hline & MARÇO & $* * 691$ & 50 & $\mathrm{R} \$ 39.564,00$ & 2 \\
\hline & MARÇO & $* * 695$ & 50 & $\mathrm{R} \$ 85.814,00$ & 2 \\
\hline & MARÇO & $* * 764$ & 8 & $\mathrm{R} \$ 5.760,00$ & 2 \\
\hline & MARÇO & $* * 774$ & 30 & $\mathrm{R} \$ 15.638,40$ & 1 \\
\hline \multirow{4}{*}{$\begin{array}{l}\mathbf{2} \\
0 \\
0 \\
0\end{array}$} & ABRIL & $* * 088$ & 225 & $\mathrm{R} \$ 203.042,25$ & 6 \\
\hline & ABRIL & $* * 085$ & 150 & $\mathrm{R} \$ 147.000,00$ & 5 \\
\hline & ABRIL & $* * 086$ & 75 & $\mathrm{R} \$ 89.250,00$ & 5 \\
\hline & ABRIL & $* * 087$ & 300 & $\mathrm{R} \$ 222.951,00$ & 5 \\
\hline
\end{tabular}

Fonte: Adaptado de Werkema (2013)

Foi identificada a quantidade de dias que cada uma das 173 ordens de produção ficou aguardando as chegadas das caixas para finalizar o processamento (FIGURA 1). A disso, foi 
possível mensurar que $60 \%$ das ordens de produção paradas por falta de embalagem permaneceram até cinco dias aguardando as caixas para serem finalizadas e faturadas.

Figura 1 - Quantidade de dias que as OPs ficaram paradas por período

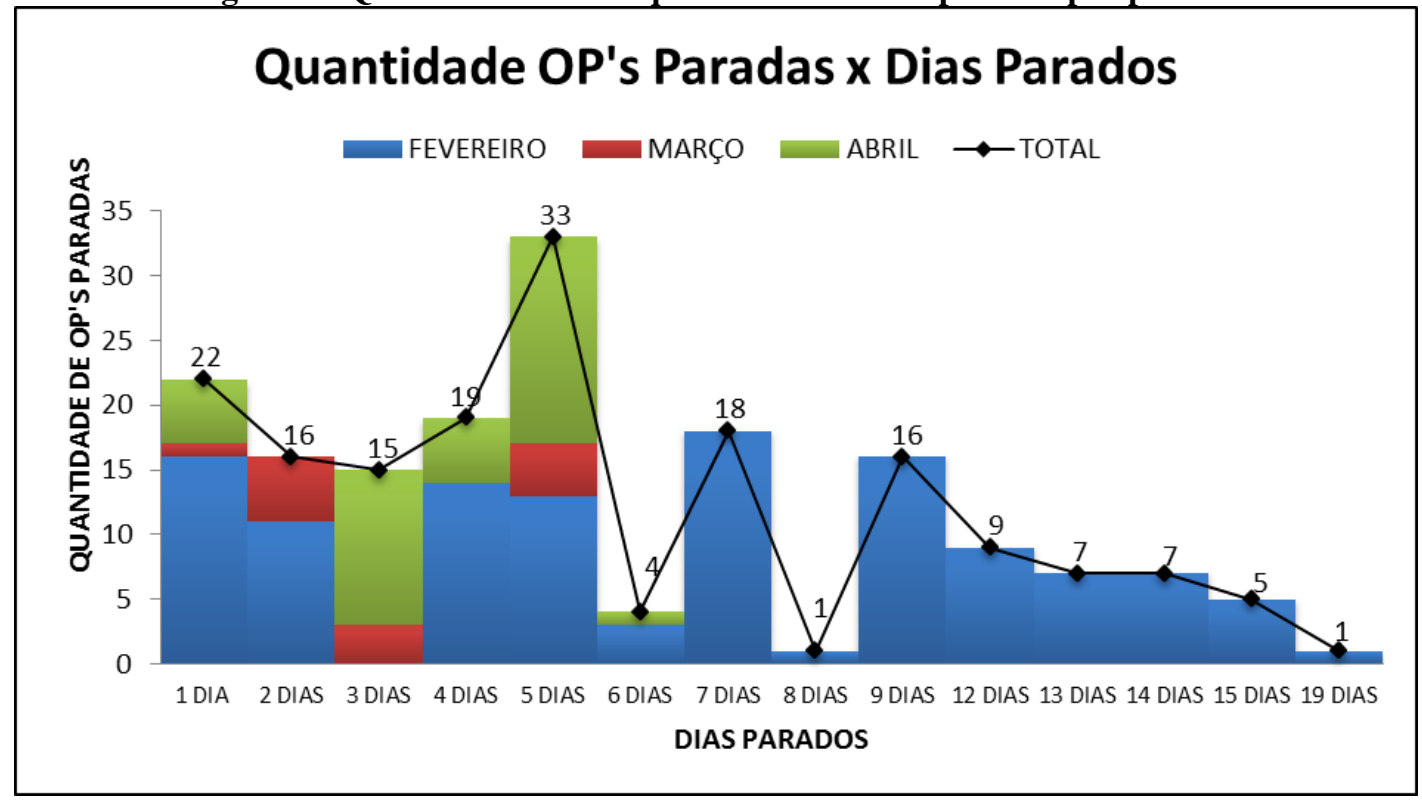

Fonte: $\mathrm{O}$ autor

Também foram identificados os valores para faturamento de cada OP parada, avaliando o tempo decorrido entre a data de entrada para a produção e a data de disponibilização para o estoque de produto acabado. Utilizou-se a técnica de análise econômica, levantando o valor de faturamento dos pedidos para mensurar o montante que ficou parado no processo de produção. Estes dados estão representados por um Gráfico de Pareto (FIGURA 2), o qual permitiu evidenciar o valor monetário total dos pedidos que ficaram aguardando os insumos de embalagem para serem finalizados e faturados pela empresa.

Figura 2 - Valor Total dos Pedidos Parados por Período

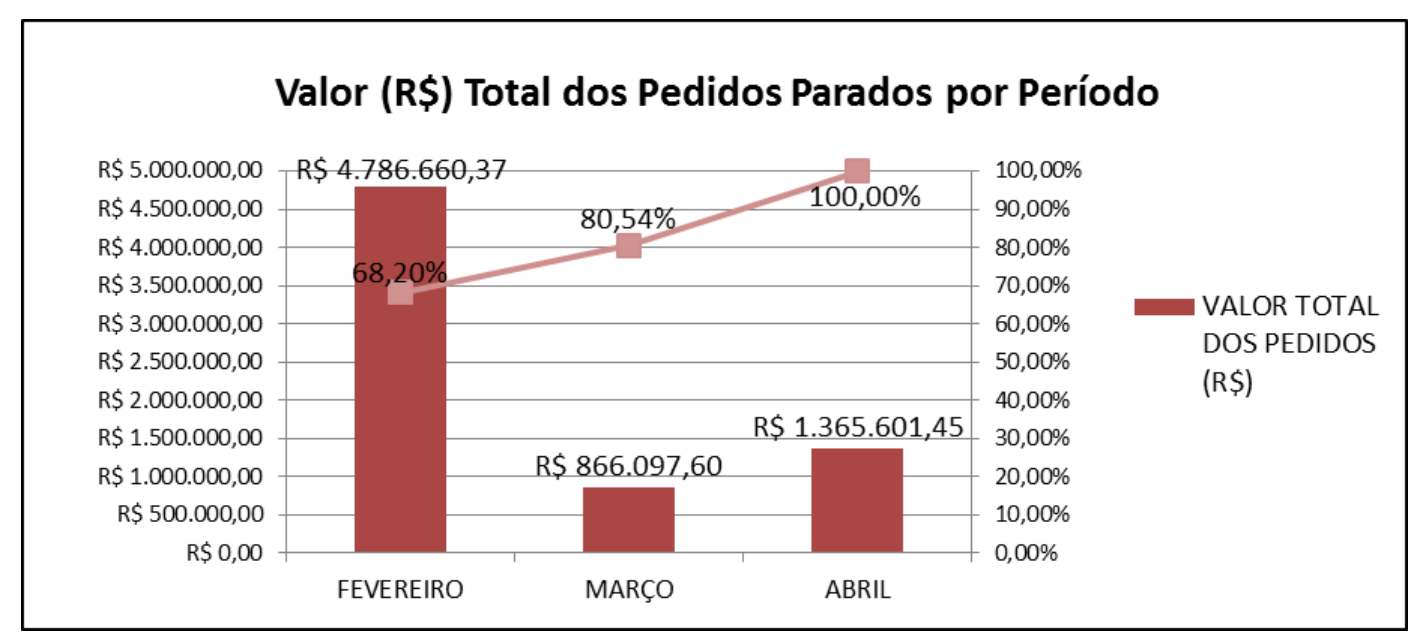

Fonte: $\mathrm{O}$ autor 
Os custos de aquisição dos cinco tipos de caixas para cada período estão representados na Tabela 1, tendo sido mensurados por meio da multiplicação do custo unitário da caixa pela quantidade mensal adquirida.

Tabela 1 - Custos de aquisição das caixas no cenário atual

\begin{tabular}{|c|c|c|c|c|c|c|c|}
\hline \multirow[b]{2}{*}{ CAIXA } & \multirow[b]{2}{*}{$\begin{array}{c}\text { CUSTO } \\
\text { UNITÁRIO }\end{array}$} & \multicolumn{2}{|c|}{ FEVEREIRO } & \multicolumn{2}{|c|}{ MARÇO } & \multicolumn{2}{|c|}{ ABRIL } \\
\hline & & $\begin{array}{c}\text { QUANTIDADE } \\
\text { MENSAL } \\
\text { ADQUIRIDA }\end{array}$ & $\begin{array}{l}\text { CUSTO } \\
\text { TOTAL }\end{array}$ & $\begin{array}{c}\text { QUANTIDADE } \\
\text { MENSAL } \\
\text { ADQUIRIDA }\end{array}$ & $\begin{array}{l}\text { CUSTO } \\
\text { TOTAL }\end{array}$ & $\begin{array}{c}\text { QUANTIDADE } \\
\text { MENSAL } \\
\text { ADQUIRIDA }\end{array}$ & $\begin{array}{l}\text { CUSTO } \\
\text { TOTAL }\end{array}$ \\
\hline TIPO A & $\mathrm{R} \$ 3,86$ & 9.072 & $\mathrm{R} \$ 35.017,92$ & 6.947 & $\mathrm{R} \$ 26.815,42$ & 6.674 & $\mathrm{R} \$ 25.761,64$ \\
\hline TIPO S & $\mathrm{R} \$ 3,64$ & 4.673 & $\mathrm{R} \$ 17.009,72$ & 4.754 & $\mathrm{R} \$ 17.304,56$ & 6.041 & $\mathrm{R} \$ 21.989,24$ \\
\hline TIPO N & $\mathrm{R} \$ 1,98$ & 3.307 & $R \$ 6.547,86$ & 5.510 & $\mathrm{R} \$ 10.909,80$ & 5.070 & $R \$ 10.038,60$ \\
\hline TIPO H & $\mathrm{R} \$ 5,33$ & 207 & $\mathrm{R} \$ 1.103,31$ & 619 & $\mathrm{R} \$ 3.299,27$ & 1.000 & $R \$ 5.330,00$ \\
\hline TIPO PH & $\mathrm{R} \$ 7,00$ & 1.524 & $\mathrm{R} \$ 10.668,00$ & 3.210 & $\mathrm{R} \$ 22.470,00$ & 7.448 & $\mathrm{R} \$ 52.136,00$ \\
\hline TOTAI & MENSAL & 18.783 & R\$ 70.346,81 & 21.040 & $\mathrm{R} \$ \mathbf{8 0 . 7 9 9 , 0 5}$ & 26.233 & $\mathrm{R} \$ \mathbf{1 1 5 . 2 5 5 , 4 8}$ \\
\hline
\end{tabular}

Fonte: $\mathrm{O}$ autor

\subsection{Fase Analyse}

A partir dos dados da etapa anterior, foi possível identificar os tipos de insumos e as quantidades de caixas que resultaram nas paradas do processo de embalagem dos computadores e o prejuízo financeiro provocado pelas OP's paradas. No mês de Fevereiro, as 121 OPs paradas representaram $\mathrm{R} \$ 4.786 .660,37$, correspondendo a $68,20 \%$ do montante analisado. Já em Março, somente 13 OP's ficaram paradas por falta de insumos, mas mesmo assim significaram $\mathrm{R} \$ 866.097,60$, representando $12,34 \%$ do montante. Por fim, no período de Abril, houve 39 OP's paradas que representaram $\mathrm{R} \$ 1.365 .601,45$, correspondendo a $19,46 \%$ do montante analisado.

A quantificação das perdas econômicas ocasionadas pela quantidade de dias parados de cada OP foi arbitrada por meio da aplicação de taxa de 2,5\% a.m. sobre o valor do pedido parado por falta do insumo. No período de Fevereiro as perdas foram estimadas em $\mathrm{R} \$ 28.961,48$. Para Março, as perdas foram estimadas em $\mathrm{R} \$ 1.976,40$. Já para Abril as perdas são representadas pelo valor de $\mathrm{R} \$ 5.172,71$. O somatório das perdas dos períodos é estimado em $\mathrm{R} \$ 36.110,59$.

Após a identificação e mensuração das falhas no processo de embalagem relacionadas à falta de caixas seguiu-se para a investigação da causa raiz do problema. O brainstormig possibilitou o levantamento de causas potenciais, representadas no diagrama de causa e efeito para a falta dos insumos (FIGURA 3).

Figura 3 - Diagrama de Causa e Efeito para a falta de caixas.

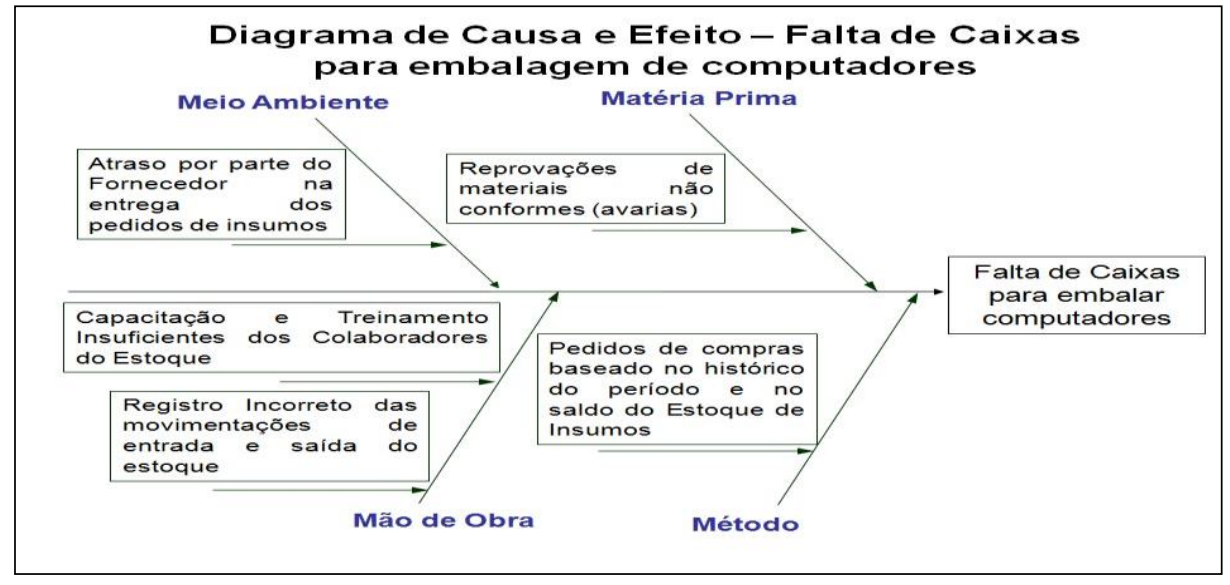

Fonte: $\mathrm{O}$ autor 
A análise da falta de caixa gerou cinco causas potenciais, sendo a causa "Pedidos de compras baseado no histórico e no saldo do Estoque de Insumos" foi identificada como a causa raiz para o problema.

\subsection{Fase Improve}

A partır da identıtıcação da causa raız dos problemas, decidıu-se pela utılızação de um método de previsão dos insumos para projeção de um novo cenário. Escolheu-se o método de técnica quantitativa média móvel simples (FERNANDES; GODINHO FILHO, 2010), pois a quantidade de dados referentes à utilização de insumos existentes no sistema de gestão da empresa limitava a utilização de modelos mais aprimorados, que necessitam de um maior número de dados. Para este projeto, estabeleceu-se a quantidade de $3(N)$ períodos. Como o período de análise deste projeto inicia-se no mês de Fevereiro de 2013, foram utilizados dados da demanda de caixas dos meses de Novembro e Dezembro de 2012 e de Janeiro de 2013.

Estabeleceu-se também para o projeto a adoção de estoque de segurança para os insumos, em virtude das constantes variações do mercado. A proposição desta ação exigiu a definição de um nível de serviço e, firmou-se o valor de 95\%. Fernandes e Godinho Filho (2010) estabelecem uma relação entre fator de segurança, denominado de $n$, e o desvio padrão ( ) na determinação do estoque de segurança: . O valor do fator de segurança para o

nível de serviço de $95 \%$ é de $n=1,645$. A Tabela 2 apresenta a integração dos dados de previsão de demanda, demanda real, desvio padrão da demanda, fator de segurança e estoque de segurança.

Tabela 2 - Previsão de demanda das caixas no cenário projetado

\begin{tabular}{|c|c|c|c|c|c|c|}
\hline \multicolumn{7}{|c|}{ CAIXA TIPO A } \\
\hline Período & $\begin{array}{c}\text { Previsão de } \\
\text { Demanda }\end{array}$ & $\begin{array}{l}\text { Desvio Padrão } \\
(\sigma)\end{array}$ & $\begin{array}{c}\text { Fator de } \\
\text { Segurança (n) }\end{array}$ & $\begin{array}{c}\text { Estoque de } \\
\text { Segurança (s) }\end{array}$ & $\begin{array}{c}\text { Previsão + Estoque de } \\
\text { Segurança }\end{array}$ & Demanda Real \\
\hline $\mathrm{fev} / 13$ & 5.327 & 2.270 & 1,645 & 3.735 & 9.061 & 3.613 \\
\hline $\mathrm{abr} / 13$ & 4.335 & 1.790 & 1,645 & 2.945 & 7.279 & 3.960 \\
\hline \multicolumn{7}{|c|}{ CAIXA TIPO S } \\
\hline Período & $\begin{array}{c}\text { Previsão de } \\
\text { Demanda }\end{array}$ & $\begin{array}{l}\text { Desvio Padrão } \\
(\sigma)\end{array}$ & $\begin{array}{c}\text { Fator de } \\
\text { Segurança (n) }\end{array}$ & $\begin{array}{c}\text { Estoque de } \\
\text { Segurança (s) }\end{array}$ & $\begin{array}{c}\text { Previsão + Estoque de } \\
\text { Segurança }\end{array}$ & Demanda Real \\
\hline fev/13 & 3.798 & 627 & 1,645 & 1.031 & 4.828 & 4.485 \\
\hline mar/13 & 4.261 & 616 & 1,645 & 1.014 & 5.275 & 3.758 \\
\hline $\mathrm{abr} / 13$ & 4.179 & 542 & 1,645 & 892 & 5.071 & 4.474 \\
\hline \multicolumn{7}{|c|}{ CAIXA TIPO N } \\
\hline Período & $\begin{array}{c}\text { Previsão de } \\
\text { Demanda }\end{array}$ & $\begin{array}{l}\text { Desvio Padrão } \\
(\sigma)\end{array}$ & $\begin{array}{c}\text { Fator de } \\
\text { Segurança (n) }\end{array}$ & $\begin{array}{c}\text { Estoque de } \\
\text { Segurança (s) }\end{array}$ & $\begin{array}{c}\text { Previsão + Estoque de } \\
\text { Segurança }\end{array}$ & Demanda Real \\
\hline Período & $\begin{array}{c}\text { Previsão de } \\
\text { Demanda }\end{array}$ & $\begin{array}{l}\text { Desvio Padrão } \\
(\sigma)\end{array}$ & $\begin{array}{c}\text { Fator de } \\
\text { Segurança (n) }\end{array}$ & $\begin{array}{c}\text { Estoque de } \\
\text { Segurança (s) }\end{array}$ & $\begin{array}{c}\text { Previsão + Estoque de } \\
\text { Segurança }\end{array}$ & Demanda Real \\
\hline fev/13 & 383 & 153 & 1,645 & 251 & 634 & 345 \\
\hline mar/13 & 321 & 126 & 1,645 & 207 & 528 & 300 \\
\hline abr/13 & 346 & 114 & 1,645 & 188 & 533 & 467 \\
\hline \multicolumn{7}{|c|}{ CAIXA TIPO PH } \\
\hline Período & $\begin{array}{c}\text { Previsão de } \\
\text { Demanda }\end{array}$ & $\begin{array}{l}\text { Desvio Padrão } \\
(\sigma)\end{array}$ & $\begin{array}{c}\text { Fator de } \\
\text { Segurança (n) }\end{array}$ & $\begin{array}{c}\text { Estoque de } \\
\text { Segurança (s) }\end{array}$ & $\begin{array}{c}\text { Previsão + Estoque de } \\
\text { Segurança }\end{array}$ & Demanda Real \\
\hline fev/13 & 832 & 573 & 1,645 & 942 & 1.774 & 510 \\
\hline mar/13 & 817 & 494 & 1,645 & 813 & 1.630 & 2.920 \\
\hline abr/13 & 1.293 & 1.060 & 1,645 & 1.744 & 3.037 & 6.060 \\
\hline
\end{tabular}

Fonte: $\mathrm{O}$ autor 
A análise da Tabela 2 identifica três situações em que a demanda real foi superior ao previsto. No mês de Fevereiro, a demanda real para a caixa TIPO N é 645 unidades superior ao previsto. Para o mês de Março, a caixa TIPO PH foi prevista 1.290 unidades a menos do que o necessário. Já em Abril, tem-se 3.023 unidades a menos do que a demanda real para a caixa TIPO PH.

Seguindo o mesmo procedimento anterior, estimou-se o valor monetário total das OP's para as situações em que a quantidade prevista é inferior a demanda real. O cálculo das perdas econômicas no cenário projetado é realizado utilizando a quantidade média de dias parados por falta de insumo (5 dias - ver Seção 4.2), com a taxa de 2,5\% a.m, também utilizada na Seção 4.3 para mensurar as perdas no cenário atual. O Quadro 5 apresenta a perda resultante do atraso do faturamento dos pedidos embalados com as caixas TIPO PH e TIPO $\mathrm{N}$, visto que as situações projetadas de falta de caixas são relacionadas somente aos dois modelos.

Quadro 4- Quantificação da perda resultante dos pedidos parados por falta de caixa

\begin{tabular}{|c|c|c|c|c|}
\hline CAIXA & $\begin{array}{c}\text { QUANTIDADE } \\
\text { TOTAL DE } \\
\text { PRODUTOS } \\
\text { PARADOS }\end{array}$ & $\begin{array}{c}\text { VALOR UNITÁRIO } \\
\text { MÉDIO DO } \\
\text { PRODUTO }\end{array}$ & $\begin{array}{c}\text { VALOR TOTAL DOS } \\
\text { PEDIDOS PARADOS }\end{array}$ & $\begin{array}{c}\text { PERDAS } \\
\text { RESULANTES NO } \\
\text { ATRASO DO } \\
\text { FATURAMENTO }\end{array}$ \\
\hline TIPO N & 645 & $\mathrm{R} \$ 1.049,76$ & $\mathrm{R} \$ 677.095,20$ & $\mathrm{R} \$ 2.821,23$ \\
\hline TIPO PH & 4313 & $\mathrm{R} \$ 882,99$ & $\mathrm{R} \$ 3.808 .335,87$ & $\mathrm{R} \$ 15,868,06$ \\
\cline { 4 - 5 } & \multicolumn{2}{|r}{} & $\mathrm{R} \$ 4485.431,07$ & $\mathrm{R} \$ 18,689,29$ \\
\hline
\end{tabular}

Fonte: $\mathrm{O}$ autor

Além disso, também foi contabilizado o custo de aquisição de todas as quantidades de embalagens dos produtos previstas pelo método de previsão de demanda. Os resultados indicam o custo para a manutenção do estoque de embalagens projetado a partir da implementação das melhorias (TABELA 2).

Tabela 3 - Custos de aquisição das caixas no cenário projeto pelo método de previsão

\begin{tabular}{|c|c|c|c|c|c|c|c|}
\hline \multirow[b]{2}{*}{ CAIXA } & \multirow[b]{2}{*}{$\begin{array}{c}\text { CUSTO } \\
\text { UNITÁRIO }\end{array}$} & \multicolumn{2}{|c|}{ FEVEREIRO } & \multicolumn{2}{|c|}{ MARÇO } & \multicolumn{2}{|c|}{ ABRIL } \\
\hline & & $\begin{array}{c}\text { PREVISÃO + } \\
\text { ESTOQUE } \\
\text { SEGURANÇA }\end{array}$ & $\begin{array}{l}\text { CUSTO } \\
\text { TOTAL }\end{array}$ & $\begin{array}{c}\text { PREVISÃO + } \\
\text { ESTOQUE } \\
\text { SEGURANÇA }\end{array}$ & $\begin{array}{l}\text { CUSTO } \\
\text { TOTAL }\end{array}$ & $\begin{array}{c}\text { PREVISÃO + } \\
\text { ESTOQUE } \\
\text { SEGURANÇA }\end{array}$ & $\begin{array}{l}\text { CUSTO } \\
\text { TOTAL }\end{array}$ \\
\hline TIPOA & $\mathrm{R} \$ 3,86$ & 9.061 & $\mathrm{R} \$ 34.977,38$ & 7.243 & $\mathrm{R} \$ 27.957,18$ & 7.279 & $\mathrm{R} \$ 28.098,63$ \\
\hline TIPOS & $\mathrm{R} \$ 3,64$ & 4.828 & $\mathrm{R} \$ 17.575,00$ & 5.275 & $\mathrm{R} \$ 19.201,38$ & 5.071 & $\mathrm{R} \$ 18.458,31$ \\
\hline TIPO N & $\mathrm{R} \$ 1,98$ & 2.946 & $\mathrm{R} \$ 5.832,72$ & 4.355 & $\mathrm{R} \$ 8.623,47$ & 4.816 & $\mathrm{R} \$ 9.535,95$ \\
\hline TIPO H & $\mathrm{R} \$ 5,33$ & 634 & $\mathrm{R} \$ 3.380,23$ & 528 & $\mathrm{R} \$ 2.816,71$ & 533 & $\mathrm{R} \$ 2.842,46$ \\
\hline TIPO PH & $\mathrm{R} \$ 7,00$ & 1.774 & $\mathrm{R} \$ 12.414,53$ & 1.630 & $\mathrm{R} \$ 11.409,39$ & 3.037 & $\mathrm{R} \$ 21.261,41$ \\
\hline \multicolumn{2}{|c|}{ TOTAL MENSAL } & 19.243 & R\$74.179,86 & 19.032 & $\mathrm{R} \$ 70.008,14$ & 20.737 & $\mathrm{R} \$ \mathbf{8 0 . 1 9 6 , 7 7}$ \\
\hline
\end{tabular}

Fonte: $\mathrm{O}$ autor

\subsubsection{Comparação entre os cenários atual e projetado}

Foram realizadas duas comparações entre o cenário atual e o projetado com as melhorias. Para tanto, foi avaliado o custo de manutenção do estoque na situação atual e para a projetada a partir da implementação do método de previsão de demanda. Além disso, também foi calculado o custo do atraso do faturamento dos pedidos em razão da falta de embalagem no estoque. Utilizando estes dois indicadores foi possível constatar uma significativa economia (QUADRO 6). 
Quadro 6- Comparativo entre a situação atual e a projetada

\begin{tabular}{|l|c|c|}
\cline { 2 - 3 } \multicolumn{1}{c|}{} & $\begin{array}{l}\text { Custo do Estoque (valor das } \\
\text { unidades } \\
\text { embalagens no estoque) }\end{array}$ & $\begin{array}{l}\text { Perda resultante do não faturamento } \\
\text { armazenadas falta de embalagem } \\
\text { por }\end{array}$ \\
\hline Situação atual & $\mathrm{R} \$ 266.401,34$ & $\mathrm{R} \$ 36.110,59$ \\
\hline $\begin{array}{l}\text { Situação } \\
\text { projetada }\end{array}$ & $\mathrm{R} \$ 224.384,77$ & $\mathrm{R} \$ 18.689,29$ \\
\hline Redução & $-15,77 \%$ & $-48,24 \%$ \\
\hline \multicolumn{2}{|c|}{ Fonte: O autor }
\end{tabular}

Ao contabilizar o custo das caixas faltantes no estoque da situação projetada, ainda sim se atinge uma redução de $4 \%$ do custo geral do estoque de embalagens. Nas três situações em que o método de previsão utilizado no cenário projetado não identificou quantidades de caixas necessárias para suprir a demanda do período são explicadas por dois fatores. $\mathrm{O}$ primeiro caso pode ser explicado pelo lançamento de um novo produto que também utiliza a caixa TIPO N. Nos meses subseqüentes, os modelos antigos foram substituídos gradativamente pelo novo produto e, por isso, não há um comportamento crescente para a demanda da caixa TIPO N. O segundo fator interfere na demanda da caixa TIPO PH nos meses de Março e Abril. Neste caso, foram registrados novos clientes para pedidos de produtos cujas configurações são atendidas por essa caixa. Os pedidos dos novos clientes representaram uma entrada de 2.250 unidades em Março e de 4.410 unidades em Abril. Para ambos os casos, uma revisão qualitativa dos volumes previstos poderia contribuir para ajustar os valores previstos (TUBINO, 2008). Além disso, a implementação de modelos mais aperfeiçoados de previsão de demanda, tais como Box Jenkins e redes neurais poderiam prever com maior segurança os volumes de consumo de insumos, reduzindo os volumes de estoque e aumentando o nível de serviço.

\subsection{Fase Control}

A etapa anterior projetou um cenário para períodos temporais passados e, conforme previsto na Carta de Projeto (Seção 4.1), a implantação deste projeto para períodos subsequentes depende de avaliação da empresa. Mesmo assim, foi realizado um plano de ação em caso de implantação do projeto proposto, utilizando a ferramenta 5W2H (QUADRO 7).

\section{Quadro 7}

\begin{tabular}{|l|l|}
\hline \multicolumn{2}{|c|}{ Objetivo: Implantar método de previsão } \\
\hline O que fazer? & Utilizar método de previsão de demanda para os insumos de embalagem \\
\hline Porque fazer? & Reduzir as paradas de produção por falta de insumos para embalar os computadores \\
\hline Onde fazer? & Planilha de Controle de Insumos \\
\hline Quem fará? & Setor de PCP \\
\hline Quando fará? & A definir \\
\hline Como fará? & Quantificar os valores utilizados de todo os insumos mês a mês \\
\hline Quando vai custar? & Custos de aquisição dos produtos dependerão da demanda de cada período \\
\hline
\end{tabular}

Fonte: $\mathrm{O}$ autor

\section{Conclusão}

A partir da necessidade de implementar a melhoria contínua nos processos este artigo teve por objetivo propor um procedimento de melhoria contínua no controle de insumos de embalagem em uma empresa de computadores utilizando o método DMAIC. A adoção do DMAIC como procedimento de melhoria, juntamente a uma série de técnicas e ferramentas utilizadas na condução do projeto, resultou na redução estimada uma redução de 48,24\% nas 
perdas causadas pelos dias parados dos pedidos estabelecer um custo de aquisição das caixas 4\% menor, para todo o período analisado. A utilização da metodologia DMAIC contribuiu para atingir aos objetivos de eliminação de perdas no processo. O suporte da metodologia DMAIC para a filosofia enxuta se apresenta como uma alternativa para operacionalizar a melhoria contínua dos processos.

\section{Referências}

CARVALHO, M.M.; PALADINI, E.P. Gestão da Qualidade: teoria e casos. Rio de Janeiro: Elsevier, 2005.

DE MAST, J.; LOKKERBOL, J. An analysis of the six sigma DMAIC method from the perspective of problem solving. International Journal of Production Economics, v. 139, p. 604-614, 2012.

ECKES, G. A revolução Seis Sigma: o método que levou a GE e outras empresas a transformar processos em lucro. Rio de Janeiro: Elsevier, 2011.

FERNADES, F.C.F.; GODINHO FILHO, M. Planejamento e controle da produção: dos fundamentos ao essencial. São Paulo: Atlas, 2010.

KUMAR, M.; ANTONY, J.; SINGH, R. K.; TIWARI, M. K.; Perry, D. Implementing the Lean Sigma framework in an Indian SME: a case study. Production Planning and Control, v.17, n.4, p.407-423, 2006.

LI, M. C.; AL-REFAIE, A.; YANG, C. DMAIC approach to improve the capability of SMT solder printing process. IEEE Transactions on Electronics Packaging Manufacturing, v. 31, n. 2 , p. $126-133,2008$.

LIN, C.; CHEN, F. F.; WAN, H.; CHEN, Y. M.; KURIGER, G. Continuous improvement of knowledge management systems using Six Sigma methodology. Robotics and ComputersIntegrated Manufacturing, v.29, p. 93-103, 2013.

LINDERMAN, K.; SCHROEDER, R. G.; ZAHEER, S.; CHOO, A.; Six Sigma: a goal theoretic perspective. Journal of Operations Management, v. 21, p. 193-203, 2003.

MATOS, J.L. Implementação de um projeto de melhorias em um processo de reação química em batelada utilizando o método DMAIC. Dissertação (Mestrado). UFRGS, 2003.

PANDE, P. S.; NEUMAN, R. P.; CAVANAGH, R.R. Estratégia Seis Sigma: Como a GE, a Motorola e outras grandes empresas estão aguçando seu desempenho. Rio de Janeiro: Qualitymark, 2001.

PARRA, P. H.; PIRES, S. R. I. Análise da gestão da cadeia de suprimentos na indústria de computadores. Gestão e Produção, v.10, n.1, p.1-15, 2003.

PEREZ-WILSON, M. Seis Sigma: Compreendendo o conceito, as implicações e os desafios. Rio de Janeiro: Qualitymark, 1999.

PROUDLOVE, N.; MOXHAM, C.; BOADEN, R. Lessons for lean in healthcare from using Six Sigma in the NHS. Public Money and Management, v.28, n.1, p.27-34, 2008.

SANTOS, A. B. Modelo de referência para estruturar o programa de qualidade Seis Sigma: proposta e avaliação. Tese (Doutorado). UFSCAR, 2006. 
SATOlO, E. G.; ANDRIETTA, J. M.; MGUEl, P. A. C.; CAlARGE, F. A. Análise da utilização de técnicas e ferramentas no programa Seis Sigma a partir de um levantamento tipo survey. Produção, v.19, n.2, p.400-416, 2009.

SERVIN, C. A. L.; SANTOS, L. C.; GOHR, C. F. Aplicação da metodologia DMAIC para a redução de perdas por paradas não programadas em uma indústria moageira de trigo. Anais... Encontro Nacional de Engenharia de Produção, Bento Gonçalves-RS, 2012.

STEPHEN, P. Application of DMAIC to integrate Lean Manufacturing and Six Sigma. Thesis Master of Science. Virginia Polytechnic Institute and State University, 2004.

TUBINO, D. F. Planejamento e controle da produção: teoria e prática. São Paulo: Atlas, 2008.

WERKEMA, C. Criando a cultura Seis Sigma. Rio de Janeiro: Qualitymark, 2002.

WERKEMA, C. Métodos PDCA e DMAIC e suas ferramentas analíticas. Rio de Janeiro: Elsevier, 2013. 